\title{
A Spectrum Decision Framework For Cognitive Radio Networks: Overview
}

\author{
${ }^{1}$ Mitul D. Patel, ${ }^{2}$ Prof. S. B. Mule, ${ }^{3}$ Dr. S. R. Ganorkar \\ Department of Electronics \& Telecommunication Engineering, Sinhgad College of Engineering, Pune-411 041 India \\ Department of Electronics \& Telecommunication Engineering, Sinhgad College of Engineering, Pune-411 041 India
}

\begin{abstract}
Cognitive radio networks are developed for solutions to spectrum inefficiency problem. In this Paper, we propose spectrum decision framework that will capable of maintaining data loss rate at lower level and total capacity at higher level. The objective of the spectrum decision framework for real time applications traffic, MVSD (Minimum Variance Based Spectrum Decision) will implement which determines the spectrum bands to minimize the capacity variance i.e. it maintains the data loss rate although the number of users increases and for best effort applications traffic, MCSD (maximum Capacity Based Spectrum Decision) will implement which decides spectrum bands to increase or maximize the total capacity. Spectrum decision framework is separate or reduces the all spectrum decision functionalities from spectrum sharing. Spectrum decision framework provides efficient bandwidth utilization.
\end{abstract}

Keywords; Cognitive radio networks, spectrum decision, spectrum resource management, minimum variance based spectrum decision, maximum capacity based spectrum decision.

\section{Introduction}

Static spectrum access techniques and growth in wireless communication resulted in consumption of the available spectrum i.e. spectrum is inefficient. Hence, dynamic spectrum access techniques such as cognitive radio technology have to be used to solve these spectrum inefficiency problems. Cognitive radio is a technology for wireless communication. The basic idea of cognitive radio networks is that the secondary user uses the licensed spectrum without affecting the transmission of primary user. A cognitive radio can enable the secondary usage of the unused portions of the licensed spectrum in order to improve spectrum utilization efficiency as well as improve the efficiency of a radio communication as a whole. Spectrum sensing, spectrum analysis and spectrum decision making are the main capabilities of the cognitive radio. Cognitive radio networks are a solution to both spectrum inefficiency and spectrum scarcity problems. However cognitive radio networks face several difficulties or problems based on the fluctuating nature of the available spectrum. To solve this problem, cognitive radio networks determine the available spectrum from the spectrum band. This process is called as spectrum sensing [3]. Spectrum sensing is used for to find the available spectrum bands and that spectrum bands are selected by networks according to the application requirements. A cognitive radio network requires the capability to decide the best spectrum band among the available bands based on the application requirements. This process is called as spectrum decision [2]. There are several different issues for cognitive radio networks to decide the spectrum band:

1. For select spectrum band, the cognitive radio network needs to consider the primary user activity and current radio conditions.

2. It needs also to consider the additional functionality spectrum sensing and spectrum sharing for reliable communication.

3. The cognitive radio networks should perform spectrum decision depends on the primary user activity and the capacity of the networks which is time varying that is changed based on application requirements [1].

Spectrum decision framework is developed by considering all decision states and applications such as real time application and best effort applications. First a capacity model is developed for to describe the primary user activity and sensing capability. After getting the available spectrum bands, most appropriate spectrum band should be selected [2].

The cognitive radio network decides appropriate spectrum bands considering with following conditions:

1. When secondary user appears in the network, it needs to be available spectrum bands for its transmission.

2. When licensed user appears in the network, secondary user moves to another available spectrum band.

3. When a selected available spectrum band is not best for secondary user then secondary user want to move to a good available spectrum band.

To consider all above conditions, the cognitive radio networks needs a spectrum decision framework. For real time applications, minimum variance based spectrum decision (MVSD) scheme is used that selects spectrum 
bands to minimize capacity variance. For best effort application, maximum capacity based spectrum decision (MCSD) scheme is used to maximize the total network capacity [1].

\section{Literature Survey}

Fast review of a spectrum decision framework for different type of traffic events such as real time and best effort application traffic events in cognitive radio network has major importance in wireless spectrum for to solve spectrum inefficiency that is it provides efficient bandwidth utilization while guaranteeing the service quality. The system that exists has a same objective to the spectrum sharing that it performs resource allocation based on service requirements. Most of the research on cognitive radio has mainly focused on efficient utilization of spectrum and power among CR users.

For spectrum allocation, a global optimization scheme is developed. Power allocation between all CR users competing the same spectrum is another important issue in spectrum sharing. Optimal power allocation scheme is proposed to achieve capacity of the fading channels.

For better understanding of cognitive radio networks, a survey on spectrum management in cognitive radio networks presents various issues in spectrum management in cognitive radio networks. The four main challenges of spectrum managements are discussed: spectrum sensing, spectrum decision, spectrum sharing, and spectrum mobility. Cognitive radio components and architectures are also discussed in this survey with spectrum management framework and transmitter detection problems such as receiver uncertainty and shadowing uncertainty. It also describes spectrum sharing that is inter-network and intra-network spectrum sharing in cognitive radio networks. A survey presents also spectrum sharing challenges such as common control channel, dynamic radio range, and spectrum unit and location information.

\section{A. Research challenges}

All of the previous work explains spectrum sharing issues where all operations performed within the same spectrum band and to adapt the fast time varying channels, they are designed as a short term operations. It is not desirable to extend existing spectrum sharing solutions designed to adapt the fast time varying channels to the long-term inter spectrum operations.

This is unique challenge in cognitive radio networks. So the objective of the spectrum decision framework is to decouple all inter spectrum functionalities from spectrum sharing.

\section{Proposed Approach}

A spectrum decision framework can provide to solution of both spectrum inefficiency and spectrum scarcity problems.

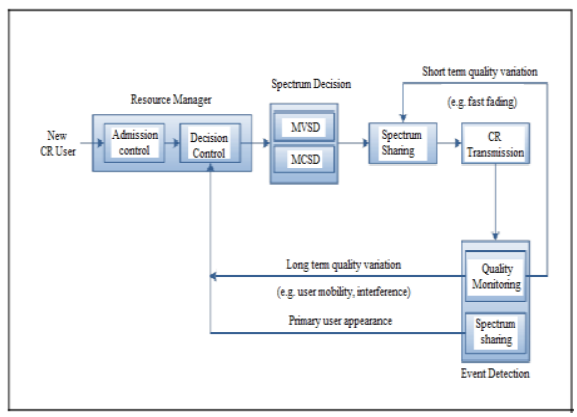

Figure 1. Block diagram of system

It determines the spectrum bands by considering requirements of application. For real time application it reduces or minimizes the capacity variance and for best effort application, it maximizes the total capacity of the network.Block diagram of spectrum decision framework for cognitive radio networks for different events such as real time application events and best effort application events is as shown in Figure 1.

\section{A. Working of the System}

Resource manager determines the incoming user is cognitive radio user or not by considering current spectrum situation. It consist admission control and decision control. If a new CR user is allowed to transmit, spectrum decision is used for assigned the proper spectrum band. Spectrum sharing is used for to coordinate the multiple accesses to avoid collisions and maximize the capacity. Event detection is used for to monitor the current spectrum band and users connections to detect decision events. When events are detected, the cognitive radio network reconfigures its resource allocation to maintain the service quality. In case of fast fading, the cognitive network reallocates resources within spectrum band through spectrum sharing. If a primary user is detected, the cognitive network switches the spectrum with the help of the resource manager and the spectrum 
decision. Cognitive radios users are used for only perform event detection. Based on this information, the base station decides on spectrum availability and performs spectrum decision.

\section{B. Resource Management}

Cognitive radio network needs a resource management scheme to coordinate the spectrum decision methods adaptively with bandwidth fluctuations. The main objectives of resource management are as follows:

- The cognitive radio network is capable to identify the new incoming secondary user without any effect on the service quality of other transmitting users.

- Cognitive radio network needs to be maintaining the service quality of transmitting users under fluctuation of the available bandwidth.

- Cognitive radio network may be required to balance the bandwidth between different application requirements.

Admission control is nothing but if cognitive radio network cannot maintain the service requirement of cognitive radio users, it should reject a new incoming cognitive radio user.Decision control schemes are used for both CR user and Primary user appearance. . If a new CR user is allowed to transmit, spectrum decision is used for assigned the proper spectrum band.

\section{Spectrum Decision for Real Time Applications}

More important parameters are considered for real time applications are delay and jitter. In real time applications, if packets are not arrived within a specified time then it drops the packet. Even through the network can support sustainable rate Rs on average, packets can be delayed and finally discarded in the receiver due to the variation of channel capacity i.e. if capacity below the average channel capacity Rs then it discards the packets. So here we check the performance of the system in terms of number of users versus data loss rate i.e. number of packets are discarded is very less although number of users increases. Delay factors uniquely introduced by CR networks can directly lead to data losses.

\section{Minimum Variance Based Spectrum Decision:}

Real time applications need to satisfy strict service requirements such as delay and sustainable rates. However problem is how to maximize the total network capacity. For that it is essential to guarantee the service quality of real-time applications with minimum spectrum resources. Thus the spectrum decision problem can be formulated as an optimization to minimize bandwidth utilization subjects to constraints on the sustainable rate, data loss rate and number of transceivers. So we introduce a three stage spectrum decision method as follows:

- Spectrum Selection: The network chooses spectrum bands with a lower primary user activity from the view of data loss rate caused by delay. Channel quality needs to be considered in spectrum decision for network capacity. Thus to maintains service quality and achieve the maximum network capacity, cognitive radio user selects spectrum bands according to the linear integer optimization.

Since real time application requires much strict service requirement then best effort application, they have a higher priority for resource allocation. Thus available bandwidth includes the portion of spectrum currently used by best effort application as well as the unused portion of the spectrum.

- Resource Allocation: In this step the CR network determines the bandwidth of the selected spectrum bands to meet the constraints both sustainable rate and target data loss rate. To allocate the bandwidth properly, we derive the total capacity and data loss rate of user.

Unlike the capacity, data loss rate cannot be directly used for the optimization. However, since the variance of the total capacity is proportional to the data loss rate, we can use variance for resource allocation instead of data loss rate. Based on capacity variance, the CR network determines optimal bandwidth of the selected bands to minimize the variance of total capacity.

- QoS Checkup: Optimization is based on the minimum variance, which guarantees the minimum data loss rate but may not satisfy the target loss rate. If the expected data loss rate is higher than target loss rate after optimization, we need to perform following approach to satisfy the target loss rate:

- Aggressive Approach: Cognitive radio network tries to find the proper spectrum bands to meet the service requirement without considering bandwidth efficiency. To this end, the selected band having the highest primary user activity needs to be replaced by the one with the highest, among the unselected bands that have a lower PU activity than the original one. In this approach it simply increases the bandwidth. If CR user cannot find proper spectrum band in this approach than it switches to the conservative approach.

- Conservative Approach: In this approach, real time applications assume to support multiple sustainable rates and to adjust their rate adaptively. Thus in this approach it reduces the sustainable rates to a one step lower rate instead of increasing the bandwidth.

Both approaches are applied in spectrum decision combining with resource management. 


\section{Spectrum Decision for Best Effort Application}

The objective of spectrum decision for best effort application is to maximize the network capacity.

\section{Maximum Capacity Based Spectrum Decision:}

Cognitive radio network has to perform the spectrum decision over all current transmission at every decision event which requires a high computational complexity for the maximum capacity. Resource reallocation leads to the spectrum switching of the multiple users at the same time results in quality degradation. So we used maximum capacity based spectrum decision for best effort applications. If current resource allocation is optimal, spectrum decision to maximize the network capacity can be simplified as the selection problem to choose spectrum band so that the decision gain can be maximized. The decision gain can be defined as the sum of the difference between capacity gain and capacity loss caused by the addition of new user.

For multiple selections the cognitive radio network first determines the order of the spectrum decision, and then chooses a spectrum band for each user as follows:

- Each cognitive radio user finds a candidate spectrum band with the highest decision gain if CR user losses its spectrum band.

- Though the optimization, CR user with the highest decision gain is assigned to the spectrum.

- Then cognitive radio network updates the current bandwidth allocation and repeats the MCSD for the remaining user, according to the optimization results.

\section{Application}

Spectrum decision framework is used for efficient utilization of bandwidth while satisfying the service requirements in wireless communication.

\section{Conclusion}

Framework for spectrum decision is used for to determine a set of spectrum bands by considering the channel and application requirements in the cognitive radio networks. MVSD scheme is developed for determines the spectrum bands to minimize the capacity variance for real time applications and MCSD scheme is developed for select the spectrum bands to maximize the total capacity for best effort applications. Simulation result shows that the spectrum decision framework provides efficient bandwidth utilization while guaranteeing the service quality in terms of different parameters such as data loss rate and total capacity or average user capacity.

\section{References}

[1] Won-Yeol Lee, Student Member, IEEE, and Ian F. Akyildiz, Fellow, IEEE, "A Spectrum Decision Framework for Cognitive Radio Networks", IEEE Transactions on Mobile Computing, vol. 10, no. 2, Feb. 2011.

[2] I.F. Akyildiz, W.-Y. Lee, M.C. Vuran, and S. Mohanty, "A Survey on Spectrum Management in Cognitive Radio Networks," IEEE Comm. Magazine, vol. 46, no. 4, pp. 40-48, Apr. 2008.

[3] W.-Y. Lee and I.F. Akyildiz, "Optimal Spectrum Sensing Framework for Cognitive Radio Networks," IEEE Trans. Wireless Comm., vol. 7 , no. 10 , pp. 3845-3857, Oct. 2008.

[4] L. Cao and H. Zheng, "Distributed Rule-Regulated Spectrum Sharing," IEEE J. Selected Area in Comm., vol. 26, no. 1, pp. 130-145, Jan. 2008.

[5] W.-Y. Lee and I.F. Akyildiz, "Spectrum-Aware Mobility Management in Cognitive Radio Cellular Networks," IEEE Transactions on Mobile Computing, vol. 11, no. 4, Apr. 2012.

[6] C. Chou, S. Shankar, H. Kim, and K.G. Shin, "What and How Much to Gain by Spectrum Agility?" IEEE J. Selected Areas in Comm., vol. 25, no. 3, pp. 576-588, Apr. 2007.

[7] R. Etkin, A. Parekh, and D. Tse, "Spectrum Sharing for Unlicensed Bands," IEEE J. Selected Areas in Comm., vol. 25, no. 3, pp. 517528, Apr. 2007 